\title{
Distúrbios da hemostasia em crianças portadoras de cardiopatias congênitas
}

\section{Hemostatic defects in children with congenital heart disease}

Tais S. Sena

Silvio R. Pinheiro Filho

Isa M. Lyra ${ }^{2}$

\begin{abstract}
As cardiopatias congênitas apresentam elevada prevalência no Brasil, sendo responsáveis por morbi-mortalidade importante, principalmente em menores de 5 anos de idade. Relacionam-se a um perfil complexo de alterações hemostáticas, predispondo tanto a eventos trombóticos como hemorrágicos, e cujo conhecimento é fundamental para o manejo adequado de cada paciente. O presente artigo objetiva descrever as alterações da coagulação relacionadas às cardiopatias congênitas, cianogênicas ou não, em menores de 18 anos de idade, submetidos ou não a procedimentos cirúrgicos. Rev. Bras. Hematol. Hemoter. 2010;32(2):149-154.
\end{abstract}

Palavras-chave: Cardiopatia; coagulação sanguínea; trombose.

\section{Introdução}

Estima-se que as malformações congênitas incidam em $2 \%$ a $3 \%$ dos nascidos vivos, estando em segundo lugar entre as causas de mortalidade infantil no Brasil, e em terceiro lugar quando considerada a mortalidade em menores de cinco anos. ${ }^{1}$ Dentre as anomalias congênitas graves, as do coração e grandes vasos são as mais frequentes, acometendo entre 2 a $10 / 1000$ nascidos vivos. ${ }^{2}$

No Brasil, em 1997, segundo estudo conduzido em Pernambuco, por Arruda et al., as anormalidades cardiovasculares foram responsáveis por 39,4\% de todas os óbitos por malformações. ${ }^{1}$ No estado do Paraná, a incidência estimada de malformações congênitas cardiovasculares é de 4/1000. ${ }^{2}$ As cardiopatias mais comuns, de acordo com estudo populacional conduzido nesse estado, foram a comunicação interventricular $(28,3 \%)$ e defeito atrioventricular $(8,1 \%){ }^{3}$

Os pacientes com cardiopatias congênitas exibem um desequilíbrio entre as atividades pró e anticoagulante, predispondo a eventos tromboembólicos, assim como eventos hemorrágicos, estes principalmente relacionados às cirurgias com circulação extracorpórea (CEC). ${ }^{4}$ Diante da alta prevalência de tais patologias, e da maior necessidade de intervenções cirúrgicas, alterações complexas no perfil de coagulação desses pacientes têm sido cada vez mais observadas, sendo enfoque crescente de vários estudos nas últimas décadas.

O objetivo do presente artigo é descrever as alterações hemostáticas que ocorrem nos pacientes com cardiopatias congênitas, submetidos ou não a intervenções cirúrgicas, à luz da literatura vigente.

\section{Material e Método}

Foi realizada pesquisa no banco de dados PubMed, através da relação entre os seguintes unitermos: congenital heart disease, cyanotic congenital heart defects, coagulation defects, hemostatic defects, coagulation factors deficiences, cardiopulmonary bypass, hemodilution, para artigos publicados em língua inglesa e portuguesa, de ensaios conduzidos em seres humanos e artigos de revisão, sem restrições quanto ao ano de publicação, sendo selecionados 28 artigos, incluídos na revisão.

\footnotetext{
${ }_{2}^{1}$ Médica Residente em Hematologia Pediátrica do Hospital Santo Antônio/Obras Sociais Irmã Dulce - Salvador-BA.

${ }^{2}$ Gerente da Hematologia Pediátrica da Universidade Federal da Bahia; Coordenadora da Agência Transfusional do Hospital Ana Neri; Preceptora da Residência Médica de Hematologia do Hospital Santo Antônio/Obras Sociais Irmã Dulce - Salvador-BA.
}

Hospital Santo Antônio/Obras Sociais Irmã Dulce; Hospital Ana Neri - Salvador-BA.

Correspondência: Tais Soares Sena

Rua das Jandaias, 74, apto. 1203 - Imbui

41720-090 - Salvador-BA - Brasil

E-mail:taisssena@hotmail.com

Doi: $10.1590 /$ S1516-84842010005000036 


\section{Alterações hemostáticas nas cardiopatias congênitas}

O sistema de coagulação humano atua através de proteínas pró-coagulantes e anticoagulantes com objetivo de manter a hemostasia. ${ }^{5}$ Seus componentes devem atuar de forma a evitar perda excessiva de sangue e formação de trombos intravasculares, garantindo o equilíbrio funcional e regulação do fluxo sanguíneo. Atualmente, aceita-se que mecanismos hemostáticos estejam associados com três complexos enzimáticos pró-coagulantes, os quais envolvem serinoproteases dependentes de vitamina K (fatores II, VII, IX, X) associadas a cofatores (V e VIII), todos localizados em uma superfície de membrana contendo fosfolipídeos, conforme demonstrado na Figura $1 .{ }^{6}$

As anormalidades da coagulação que ocorrem em crianças com cardiopatias congênitas representam uma complexa interação entre fatores e cofatores da coagulação, podendo incluir defeitos preexistentes, bem como secundários à CEC. ${ }^{5,7}$ Nas cardiopatias congênitas, principalmente cianogênicas, observa-se um desequilíbrio destes mecanismos regulatórios, por razões não totalmente elucidadas, levando a um maior risco de tromboembolismo arterial e venoso e acidente vascular cerebral (AVC), conforme esquematizado na Figura 2. ${ }^{8,9}$ Disfunção endotelial, hiperviscosidade, coagulação intravascular disseminada e ativação

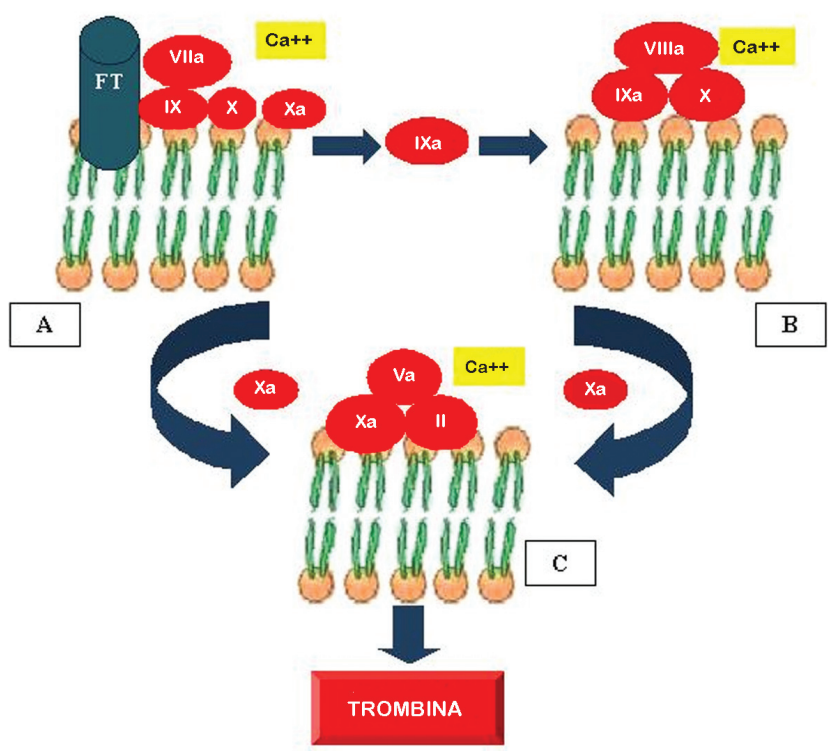

Figura 1. Representação esquemática dos complexos pró-coagulantes. A formação do coágulo de fibrina envolve complexas interações entre proteases plasmáticas e seus cofatores. Através da ligação do fator VIla ao fator tecidual (FT), ocorre ativação dos fatores IX e X. O complexo fator IXa/VIIla ativa o fator X com eficiência ainda maior, e o fator Xa forma complexo com o fator $\mathrm{Va}$, convertendo o fator II (protrombina) em fator Ila (trombina), que por proteólise converte o fibrinogênio solúvel em fibrina insolúvel. Adaptado de Rendrik F Franco. Fisiologia da coagulação, anticoagulação e fibrinólise. Medicina, Ribeirão Preto 2001;34:229-37

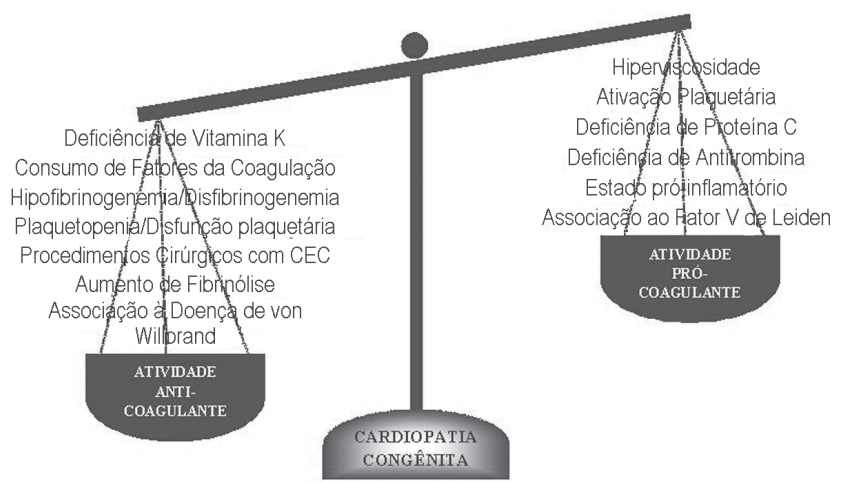

Figura 2. Desequilíbrio entre as atividades pró- e anticoagulante nas crianças portadoras de cardiopatias congênitas, principalmente cianogênicas. No exemplo, predomínio dos mecanismos prócoagulantes, predispondo a eventos tromboembólicos

plaquetária são fatores implicados na gênese de tais distúrbios. ${ }^{4,7}$

Dentre as alterações da coagulação observadas em crianças com cardiopatia congênita podem ocorrer deficiências de proteína C, fatores II, V, VII e X, plasminogênio e antitrombina, fibrinogênio, disfibrinogenemia e aumento da fibrinólise. ${ }^{7,9,10}$

Na década de 60 , Somervile et al. já observaram as alterações hemostáticas em 50 pacientes com cardiopatias congênitas (40 cianogênicas e 10 não cianogênicas), entre elas tetralogia de Fallot, atresia tricúspide, estenose pulmonar, desvio do septo interatrial e transposição de grandes artérias. Os distúrbios identificados foram hipofibrinogenemia, plaquetopenia, redução da síntese de tromboplastina, sendo esta última alteração proporcional ao nível de policitemia em pacientes com cardiopatias cianogênicas. Esses distúrbios foram relacionados a uma maior tendência hemorrágica em traumas e/ou cirurgias nessas crianças. Os autores já sugeriam que as coagulopatias observadas nos indivíduos policitêmicos pudessem ocorrer como efeito da hipoxemia crônica. ${ }^{11} \mathrm{Na}$ mesma década, desequilíbrios hemostáticos devido ao consumo de plaquetas, fatores de coagulação, e fibrinólise aumentada já eram atribuídos ao procedimento de CEC. ${ }^{12}$

$\mathrm{Na}$ década de 70, Inglis et al. estudaram os fatores de coagulação em 42 crianças com cardiopatia congênita submetidas à CEC, sendo 24 com cardiopatias cianogênicas e 18 não cianogênicas. Em comparação aos pacientes acianóticos, os cianóticos tiveram maior queda do fator VIII, o que esteve diretamente relacionado à duração da CEC. Os pacientes cianóticos que faleceram nas primeiras 72 horas após a CEC (12 pacientes) apresentaram maior queda dos níveis de fator $\mathrm{V}$, fibrinogênio e plaquetas, do que os pacientes cianóticos que sobreviveram. Nesses pacientes que faleceram, não houve recuperação dos níveis dos fatores de coagulação, como ocorreu com os demais incluídos no estudo. ${ }^{7}$ Múltiplos defeitos no mecanismo hemostático em pacientes com 
cardiopatia cianogênica foram encontrados por Henricksson et al., na mesma época, sobretudo níveis reduzidos de fatores vitamina $\mathrm{K}$ dependentes, como indicado pelos baixos fatores II, VII, e X ( $85 \%$ dos doentes) e de baixo fator IX (50\% destes). Além disso, o fator $\mathrm{V}$ apresentou diminuição em $50 \%$ dos pacientes. Tais alterações não foram corrigidas pela administração parenteral de vitamina $\mathrm{K}$, devido aos danos hepáticos por hipóxia, que comprometeu a síntese de complexo protrombínico. ${ }^{13}$

\section{O papel da disfunção endotelial}

O endotélio vascular pode sofrer lesão mecânica pelo aumento da viscosidade sanguínea e/ou hipoxemia crônica nas cardiopatias congênitas cianogênicas, levando a uma redução na síntese de trombomodulina, o que, por sua vez, compromete a atividade da proteína $\mathrm{C}$, perpetuando a disfunção endotelial (Figura 3). ${ }^{4,8}$

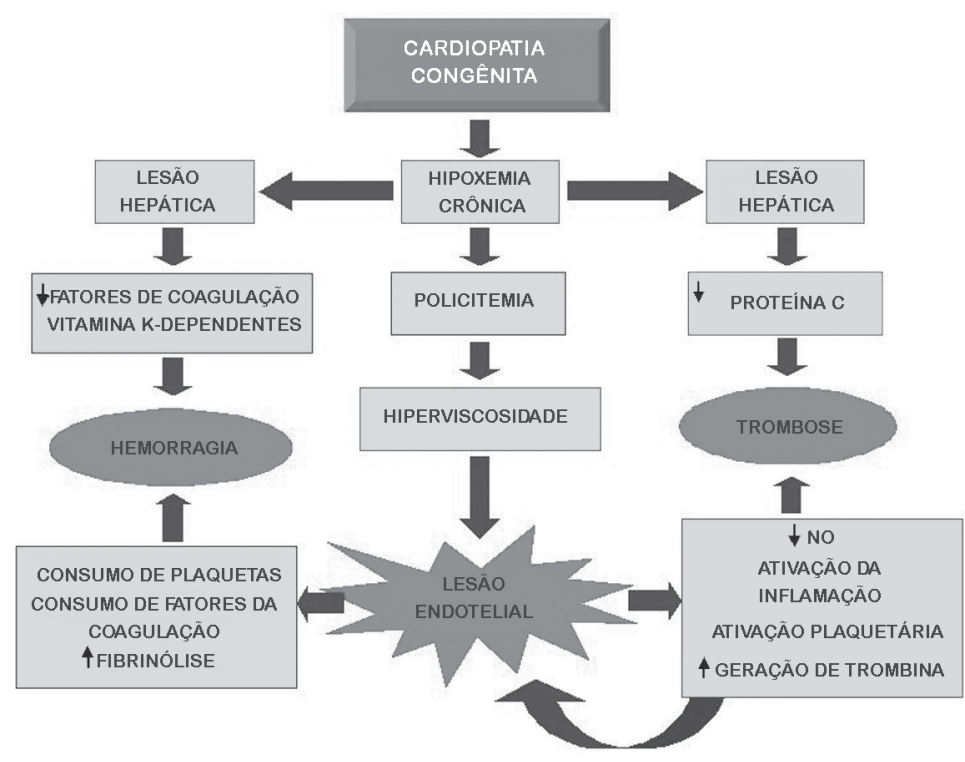

Figura 3. Mecanismos envolvidos na trombogênese e distúrbios hemorrágicos nas cardiopatias congênitas cianogênicas

A trombomodulina endotelial é um componente-chave para a via da proteína $\mathrm{C}$, atuando como uma barreira anticoagulante intrínseca entre o sangue e o endotélio vascular. Níveis reduzidos dessa substância, aliados à diminuição da síntese de óxido nítrico pelo endotélio vascular, sugerem disfunção endotelial nesses pacientes, promovendo a trombogênese. ${ }^{8}$

\section{Disfunção plaquetária}

Tais fatores lesivos contribuem ainda para a ativação plaquetária e a expressão da P-selectina em sua superfície (molécula de adesão encontrada nos grânulos plaquetários e corpos de Weibel-Palade nas células endoteliais), que pode ser um indutor direto da atividade pró-coagulante associada a doenças vasculares e trombogênicas. As plaquetas ativadas que expressam a P-selectina em sua superfície liberam seus grânulos, facilitando a adesão de outras plaquetas e neutrófilos ao endotélio, causando agregação e aumento do trombo. Em 1973, Goldschimidt registrou que a disfunção plaquetária relacionada às cardiopatias congênitas cianogênicas, particularmente a hiperagregação plaquetária, desempenharia papel central na trombogênese e coagulação intravascular disseminada nesses pacientes. ${ }^{14}$ Kajimoto et al. observaram que ocorre ativação plaquetária em pacientes com cardiopatia congênita cianogênica a despeito do uso de drogas antiplaquetárias. Os autores evidenciaram ainda aumento nos níveis do complexo trombina-antitrombina (TAT) nas crianças com eventos tromboembólicos, quando comparados aos pacientes sem eventos. Portanto, aumento da P-selectina e do TAT podem indicar maior risco de tromboembolismo nesses pacientes. ${ }^{8}$

Destaca-se como maior ativador plaquetário a trombina, que leva à produção do inibidor da via do fator tecidual. Durante a CEC, a geração acelerada de trombina em crianças portadoras de cardiopatia congênita e cianogênica confirma o estado prócoagulante relacionado à intervenção. ${ }^{10}$

\section{Estado pró-inflamatório}

A ativação dos receptores da trombina nos leucócitos aumenta a liberação de citocinas, o que contribui para o processo inflamatório. Crianças com cardiopatia congênita têm aumento da liberação de citocinas pró-inflamatorias intramiocárdicas e sistêmicas, como interleucina-1 e fator de necrose tumoral alfa, que apresentam efeito pró-trombótico pela ativação de monócitos e células endoteliais, que expressam fator tecidual. ${ }^{10}$

\section{Particularidades em menores de 6 meses de idade}

O sistema de coagulação dos lactentes menores de 6 meses de idade exibe diferenças qualitativas e quantitativas na maioria dos pró-coagulantes e inibidores. Nesses pacientes, há uma menor capacidade de gerar trombina, e de inibila, uma vez formada, efeito este exacerbado naqueles com cardiopatias congênitas submetidos à CEC..$^{15}$ Adicionalmente, destaca-se a redução dos níveis dos fatores II, V, VII, X, XI, XII e XIII da coagulação nessa faixa etária, situação agravada em cerca de $50 \%$ dos neonatos submetidos a cirurgias cardíacas com CEC. ${ }^{4}$ Estudos subsequentes corroboraram tal observação, sendo registradas, ainda, deficiências congênitas do fator XII e fator de Von Willebrand $(\mathrm{FvW})$ associadas a cardiopatias congênitas cianogênicas. ${ }^{4}$ 


\section{Resistência à proteína $\mathrm{C}$ ativada}

A proteína $\mathrm{C}$ ativada exerce um potente efeito anticoagulante ao degradar seletivamente os fatores Va e VIIIa. Nos pacientes que apresentam resistência à proteína $\mathrm{C}$ ativada, esta atividade está habitualmente reduzida. $\mathrm{Na}$ grande maioria dos casos (em mais de 95\%), essa resistência é herdada geneticamente, sendo a mutação do fator $\mathrm{V}$ mais comumente referida como fator $\mathrm{V}$ Leiden. A proteína $\mathrm{C}$ ativada neutraliza o fator $\mathrm{V}$ Leiden lentamente, levando a um estado pró-coagulante. A prevalência do fator $\mathrm{V}$ Leiden em pacientes com doença cardíaca congênita está estimada em torno de $4,5 \%$, o que, no entanto não difere da prevalência na população geral, não sendo possível concluir sobre a associação entre o fator mutante e episódios trombóticos no pósoperatório dessas crianças. ${ }^{5}$

\section{Associação a síndromes genéticas}

Vale ressaltar que algumas síndromes genéticas que estão associadas a cardiopatias congênitas podem cursar com distúrbios hematológicos, os quais podem influenciar a evolução e conduta durante os procedimentos cirúrgicos. Dentre elas, destaca-se a síndrome de Noonan, caracterizada por baixa estatura, alterações faciais, cardiopatia congênita (mais frequentemente estenose pulmonar e miocardiopatia hipertrófica), criptorquidia, anormalidades esqueléticas e diátese hemorrágica. ${ }^{16,17}$ As principais alterações relacionadas a esta síndrome são deficiências de fatores XI, VIII e XII, trombocitopenia e disfunção plaquetária, acarretando manifestações hemorrágicas em $55 \%$ dos portadores da síndrome. ${ }^{16,18}$ Pode haver ainda um padrão semelhante de deficiência de fatores da coagulação em parentes de primeiro grau de portadores da síndrome. ${ }^{17}$

\section{Distúrbios de coagulação associados aos procedimentos cirúrgicos}

O procedimento de CEC envolve importantes fases: canulação dos grandes vasos; anticoagulação; contato do sangue com superfícies artificiais; fluxo sanguíneo nãopulsátil; esfriamento ou hipotermia; contato direto do sangue com o oxigênio; cardioplegia (maioria dos casos) e parada circulatória total sob hipotermia em alguns casos (Figura 4). ${ }^{19}$

$\mathrm{O}$ processo lesivo relacionado à CEC é multifatorial e pode ser subdividido em três principais mecanismos: lesão mecânica dos elementos sanguíneos através dos circuitos, processo inflamatório pelo contato com as superfícies artificiais e alterações relacionadas à hemodiluição. ${ }^{20,21} \mathrm{~A}$ CEC promove ainda ativação da coagulação, que é bloqueada pelo uso da heparina. As complicações que podem surgir em relação a sangramento excessivo dependem da inadequação dos mecanismos da coagulação e fibrinólise ou decorrem da

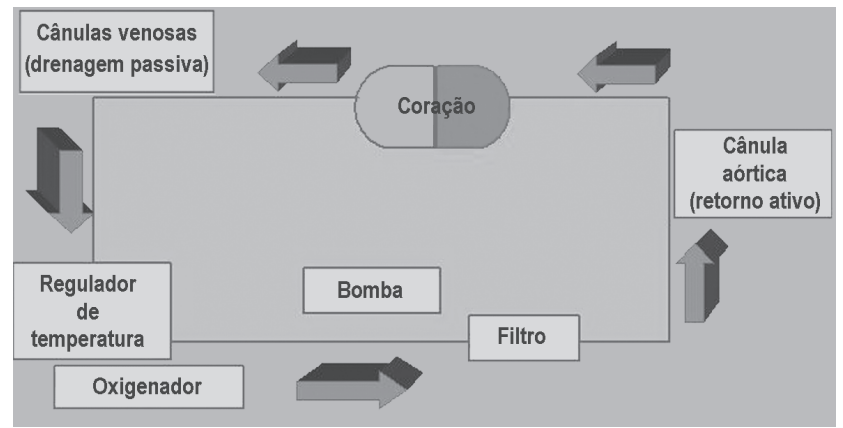

Figura 4. Representação esquemática do circuito da CEC. Adaptado de Garros D, Botta A, Piva JP, Garcia PCR. Cuidados pré e pósoperatórios em cirurgia cardíaca. In: Piva JP \& Garcia PCR. Medicina Intensiva em Pediatria. Revinter, Rio de Janeiro 2005, $1^{\text {a }}$ ed, 247-268 p

utilização do anticoagulante. Durante a CEC, ocorre hemólise de graus variáveis e as plaquetas aderem à superfície dos circuitos, formando microagregados, sendo consumidas. Há redução dos fatores de coagulação devido ao consumo e à hemodiluição. Manifestações trombóticas são menos frequentes que as hemorrágicas e mais encontradas em pacientes cianóticos e nos portadores de prótese valva. ${ }^{22}$

A hemodiluição foi introduzida por Panico e Neptune, em 1959, com o intuito de minimizar as transfusões durante as cirurgias cardíacas abertas através do uso de soluções cristaloides. Apesar da redução da necessidade transfusional, o procedimento acarreta redução da pressão coloidosmótica, com queda dos níveis de albumina em torno de $32 \%$ ao final da CEC, além da diminuição do hematócrito, aumento do sódio e da água livre nas células musculares e hemácias, e perda de potássio intracelular. Outro efeito observado é a redução dos fatores de coagulação, em média $30 \%$ a $36 \%$, com recuperação aos níveis basais em 48 horas após a CEC. ${ }^{20}$

A anticoagulação durante a CEC é necessária para prevenir a formação de trombina e consequente coagulação à medida que o sangue entra em contato com as superfícies artificiais do circuito extracorpóreo. A heparina não fracionada é administrada para inibir não apenas a formação de trombina, mas também a atividade da trombina circulante. Sua ação é mediada através de sua ligação à antitrombina, alterando sua conformação molecular, tornando-a um potente inibidor da trombina circulante. ${ }^{23}$ Os efeitos da heparina são revertidos com a administração de protamina, no entanto, o efeito residual da heparina configura entre as causas de hemorragia pós-operatória. ${ }^{19}$

A disfunção plaquetária é crucial para a hemorragia pósoperatória observada em crianças com cardiopatia congênita. Crianças com cardiopatias congênitas e, em particular, cianogênicas, tendem a apresentar defeitos intrínsecos plaquetários que resultam em prolongamento do tempo de sangramento. ${ }^{4,24}$

Já na década de 70, Maurer et al. observaram prejuízo da agregação plaquetária em $52,1 \%$ de 65 crianças com cardiopatias congênitas cianogênica e não cianogênicas, que não 
estavam recebendo drogas antiplaquetárias. Destas, $11 \%$ apresentaram sintomas hemorrágicos e $9 \%$ tempo de sangramento prolongado, apesar de plaquetometria normal. Tal disfunção foi correlacionada à hipoxemia e policitemia nos pacientes cianóticos, com gravidade proporcional ao grau dessas alterações. ${ }^{9}$

A natureza da disfunção plaquetária associada à CEC permanece não esclarecida. Diversos tipos de defeitos intrínsecos das plaquetas têm sido descritos, incluindo o aumento da P-selectina, com consequente hiperativação plaquetária, associada à degranulação, e defeitos nas GP Ib e IIbIIIa têm sido reportadas em adultos e crianças durante a CEC. $^{24}$

Quando comparados a crianças maiores de 1 ano de idade, pequenos lactentes (abaixo de 2 meses de idade) apresentam menor aumento da P-selectina induzida pela CEC e uma menor redução da glicoproteína $\mathrm{Ib}(\mathrm{GP})$, sugerindo hiporreatividade plaquetária, conferindo maior risco de hemorragia nesses pacientes. ${ }^{24}$

As plaquetas dos neonatos apresentam aspectos morfológicos específicos que afetam a função celular (menos pseudópodes, redução dos depósitos de glicogênio, estruturas microtubulares menores e menos grânulos alfa), indicando um processo maturativo idade-dependente. Devido à volemia extremamente pequena e à grande área proporcional do circuito da CEC na cirurgia cardíaca neonatal, acredita-se que, nesse grupo de pacientes, os distúrbios hemorrágicos possam ser mais severos após cirurgias. ${ }^{24}$

Outro estudo conduzido por Miller et al. estratificou as crianças portadoras de cardiopatias congênitas submetidas à $\mathrm{CEC}$ de acordo com o peso, sendo o ponto de corte $8 \mathrm{~kg}$. Eles demonstraram que crianças com menos de $8 \mathrm{~kg}$ apresentam mais coagulopatias graves, e maior necessidade transfusional de hemocomponentes dos produtos da coagulação do que aquelas maiores de $8 \mathrm{~kg}$. Por ser uma variável conhecida no pré-operatório, o peso pode ser, portanto, utilizado para antecipar o estado de coagulação após a CEC, uma vez que reflete outros fatores que influenciam o sistema de coagulação (imaturidade idade-dependente, descompensação por insuficiência cardíaca congestiva, grau de hemodiluição na CEC). ${ }^{25}$

A análise da estrutura multimérica do $\mathrm{FvW}$ em pacientes com cardiopatias congênitas submetidos a cirurgia cardíaca resultou na identificação da perda de grandes multímeros do FvW após a CEC, produzindo fenótipo semelhante ao tipo 2A da doença de von Willebrand. Tal achado excluiu perda significativa desses elementos pela adsorção através da superfície do circuito da $\mathrm{CEC}$, sendo provável que a lesão mecânica durante o procedimento e um aumento global da atividade proteolítica plasmática sejam responsáveis por essas alterações. ${ }^{26} \mathrm{~A}$ diminuição dos multímeros de alto peso molecular do $\mathrm{FvW}$ foi identificada em crianças portadoras de cardiopatias congênitas não cianogênicas, previamente à realização de cirurgias corretivas. No entanto, não está claro até que ponto tais alterações contribuem para história de sangramento ou tempos de sangramento prolongados nesses pacientes. ${ }^{27}$

\section{Alterações promovidas pelas derivações cirúrgicas}

Além das coagulopatias induzidas pela $\mathrm{CEC}$, observamse ainda alterações hemostáticas produzidas pelas derivações cirúrgicas, como, por exemplo, a cirurgia de Fontan, usada como correção definitiva para atresia tricúspide e várias formas de corações univentriculares. Um dos efeitos da circulação anormal é o aumento crônico da pressão venosa sistêmica, o que pode levar a alterações no tamanho e função do fígado, como desordens da coagulação, fibrose hepática, hepatomegalia com ou sem ascite e enteropatia perdedora de proteína. Tais anormalidades podem predispor a eventos TE (por exemplo, devido à deficiência de proteínas $\mathrm{C}$ e $\mathrm{S}$ ), prolongamento do tempo de sangramento (por deficiência de fator VII ou V). Além disso, o átrio direito, de volume aumentado, predispõe a estase e formação de trombo ${ }^{28}$

Em crianças submetidas à cirurgia de Fontan (anatomia de ventículo único, em que o retorno venoso sistêmico é desviado para a artéria pulmonar, sem utilizar o ventrículo), ${ }^{19}$ foram constatados baixos níveis de fibrinogênio, fator $\mathrm{V}$, fator VII, proteína $\mathrm{C}$, aumento da antitrombina e prolongamento da relação de normalidade internacional (RNI). ${ }^{29}$ Nieuwenhuizen et al. avaliaram o impacto da mesma cirurgia no perfil de coagulação de 24 pacientes. Apenas dois $(8,3 \%)$ apresentaram coagulograma normal no pós-cirúrgico. Anormalidades prócoagulantes foram encontradas em 15 pacientes, incluindo deficiência de proteínas $\mathrm{C}$, S, plasminogênio ou antitrombina, aumento do D-dímero, ou do complexo TAT. A alteração mais frequente foi a deficiência de plasminogênio, seguida da deficiência de proteína C. Por outro lado, anormalidades anticoagulantes foram descritas em vinte pacientes, das quais destacam-se: deficiência de fatores V, VII, aumento do tempo de protrombina (TP), alargamento do tempo de tromboplastina parcial ativada (TTPa). A mais frequente foi alargamento do TP, seguida pela deficiência dos fatores V e VII. Todos os pacientes com prolongamento do TP tinham deficiência de fator V, fator VII ou ambos, o que não teve correlação com o tempo após a primeira cirurgia ou idade do paciente. ${ }^{28}$

\section{Conclusão}

As crianças com cardiopatia congênita, particularmente as cianogênicas, exibem um desequilíbrio na maior parte dos mecanismos hemostáticos, resultando em eventos trombóticos e/ou hemorrágicos, exacerbados pela necessidade de CEC durante as cirurgias corretivas, o que dificulta o manejo desses pacientes. As coagulopatias durante e após a CEC em pacientes pediátricos podem ser muito mais complexas devido aos fatores idade e doença-dependentes, além dos 
efeitos do uso da heparina, hemodiluição e lesão dos elementos celulares durante sua passagem pelo circuito. $\mathrm{O}$ conhecimento da variedade de tais alterações é crucial para a condução adequada de cada caso em particular.

\section{Abstract}

There is a high prevalence of congenital heart diseases in Brazil, which are responsible for high morbidity and mortality, mainly in under 5-year-old children. They are related to a complex profile of hemostatic disorders that lead to thrombotic and hemorrhagic events. Thus knowledge of the diseases is imperative for the adequate management of each patient. The objective of this article is to describe coagulation abnormalities related to congenital cyanotic and noncyanotic heart diseases, in children, undergoing surgical procedures or not. Rev. Bras. Hematol. Hemoter. 2010; 32(2):149-154.

Key words: Coronary diseases; blood coagulation disorders; thrombosis.

\section{Referências Bibliográficas}

1. Arruda TAM, Amorim MMR, Souza ASR. Mortalidade determinada por anomalias congênitas em Pernambuco, Brasil, de 1993 a 2003. Rev Assoc Med Bras. 2008;54(2):122-6.

2. Miyague NI, Cardoso SM, Meyer F, et al. Estudo Epidemiológico de Cardiopatias Congênitas na Infância e Adolescência. Análise em 4.538 Casos. Arq Bras Cardiol. 2003;80(3):269-73.

3. Guitti JCS. Aspectos epidemiológicos das Cardiopatias Congênitas em Londrina, Paraná. Arq Bras Cardiol. 2000;74(5):395-399.

4. Tempe DK, Virmani S. Coagulation Abnormalities in Patients with Cyanotic Congenital Heart Disease. J Cardioth Vasc Anesth. 2002; 16(6):752-65.

5. Ong B-C, Zimmerman AA, Zapulla DZ, et al. Prevalence of factor $\mathrm{V}$ Leiden in a population of patientes with congenital heart disease. Can J Anaesth. 1998;45(12):1176-80.

6. Rendrik F Franco. Fisiologia da coagulação, anticoagulação e fibrinólise. Medicina, Ribeirão Preto. 2001;34:229-237.

7. Inglis TC, Breeze GR, Stuart J, Abrams LD, Roberts KD. Excess intravascular coagulation complicating low cardiac output. J. Clin. Path. 1975;28(1):1-7.

8. Kajimoto H, Nakazawa M, Murasaki K, Mori Y, Tanoue K, Kasanuki $\mathrm{H}$, et al. Increased thrombogenesity in patients with cyanotic congenital heart disease. Circ J. 2007;71(6):948-53.

9. Maurer HM, McCue CM, Caul J, Still WJS. Impairment in platelet aggregation in congenital heart disease. Blood. 1972; 40(2):207-16.

10. Heying R, van Oeveren W, Wilhelm S, Schumacher K, Grabitz RG, Messmer BJ, et al. Children undergoing cardiac surgery for complex cardiac defects show imbalance between pro- and anti-thrombotic activity. Crit Care. 2006;10(6):R165.

11. Somerville J, McDonald L, Edgill ME. Post-operative haemorrhage and related abnormalities of blood coagulation in cyanotic congenital heart disease. Br Heart J. 1965;27:440-8.

12. De Gabrielle G. Some aspects of haemostasis after open-heart surgery. J Clin Pathol. 1963;16:569-72.

13. Henriksson P, Varendh G, Lundström NR. Haemostatic defects in cyanotic congenital heart disease. Br Heart J. 1979;41(1):23-7.
14. Goldschimidt B. Blood coagulation and platelet abnormalities in cyanotic congenital heart-disease. Lancet. 197317;1(7803):607.

15. Guzzetta NA, Miller BE, Todd K, Szlam F, Moore RH, Brosius KK, et al. Clinical measures of heparin's effect and thrombin inhibitor levels in pediatric patients with congenital heart disease. Anesth Analg. 2006;103(5):1131-8.

16. Bertola DL, Carneiro JDA, D'Amico EA, et al. Hematological findings in Noonan syndrome. Rev Hosp Clin Fac Med S Paulo. 2003;58(1):5-8.

17. Sharland M, Patton MA, Talbot S, et al. Coagulation-factor deficiencies and abnormal bleeding in Noonan's syndrome. Lancet. 1992;339(8784):19-21.

18. van der Burgt I. Noonan Syndrome. Orphanet J Rare Dis. 2007;2:4.

19. Garros D, Botta A, Piva JP, Garcia PCR. Cuidados Pré e PósOperatórios em Cirurgia Cardíaca. In: Piva JP, Garcia PCR. Medicina Intensiva em Pediatria. Rio de Janeiro: Revinter; 2005: 247-268p.

20. Hall TS. The pathophysiology of cardiopulomonary bypass: the risks and benefits of hemodilution. Chest. 1995;107(4):1125-33.

21. Silveira FMRC, Lourenço DM, Maluf M, et al. Alterações da hemostasia em crianças submetidas a cirurgia cardíaca com circulação extracorpórea. Arq Bras Cardiol. 1998;70(1):29-35.

22. Guzzetta NA, Miller BE, Todd K, Szlam F, Moore RH, Tosone SR. An evaluation of the effects of a standard heparin dose on thrombin inhibition during cardiopulmonary bypass in neonates. Anesth Analg. 2005;100(5):1276-82.

23. Ichinose F, Uezono S, Muto R, Uchida H, Hatori F, Terui $\mathrm{K}$, et al. Platelet hyporeactivity in young infants during cardiopulmonary bypass. Anesth Analg. 1999;88(2):258-62.

24. Miller BE, Mochizuki T, Levy JH, et al. Predicting and treating coagulopathies after cardiopulmonary bypass in children. Anesth Analg. 1997;85(6):1196-202.

25. Perutelli P, Lerzo F, Calza G, Cevasco M, Mori PG. Abnormalities of plasma von Willebrand factor multimeric structure induced by extracorporeal circulation. Haematologica. 1999;84(3):287-8.

26. Gill JC, Wilson AD, Endres-Brooks J, Montgomery RR. Loss of the largest von Willebrand factor multimers from the plasma of patients with congenital cardiac defects. Blood. 1986;67 (3):758-61.

27. van Nieuwenhuizen RC, Peters M, Lubbers LJ, Trip MD, Tijssen JG, Mulder BJ. Abnormalities in liver function and coagulation profile following the Fontan procedure. Heart. 1999;82(1):40-6.

28. Chaloupecký V, Svobodová I, Hadacová I, Tomek V, Hucín B, Tláskal T, et al. Coagulation profile and liver function in 102 patients after total cavopulmonary connection at mid term follow up. Heart. 2005;91(1):73-9.

Avaliação: Editor e dois revisores externos Conflito de interesse: sem conflito de interesse

Recebido: 09/06/2009

Aceito: 06/08/2009 